\title{
Two-dimensional transverse coherence measurement of hard-x-ray beams using near-field speckle
}

\author{
Yogesh Kashyap, Hongchang Wang, and Kawal Sawhney \\ Diamond Light Source, Harwell Science and Innovation Campus, Didcot, Oxfordshire OX11 ODE, United Kingdom
}

(Received 21 May 2015; published 22 September 2015)

\begin{abstract}
Knowledge of the transverse coherence of hard $\mathrm{x}$ rays is essential, not only for understanding the source properties, but also to study the impact of $\mathrm{x}$-ray optics. However, the precise measurement of transverse coherence in the $\mathrm{x}$-ray regime is more difficult than in the visible light regime since it often involves complex experimental setups or sophisticated $\mathrm{x}$-ray optics. In this paper, we present a model-free method to measure transverse coherence properties of $x$-ray beams by using a simple phase membrane. Our method allows one to map the two-dimensional source distribution in the transverse plane by analyzing the power spectrum of x-ray near-field speckle patterns, which are collected at a single distance only. The method has been validated by performing measurements for a range of source sizes, which was achieved by varying the vertical coupling of the electron beam in the Diamond storage ring. We expect that this method will be widely used in transverse coherence measurements for both synchrotron sources and x-ray free-electron lasers.
\end{abstract}

DOI: 10.1103/PhysRevA.92.033842

PACS number(s): 42.25.Kb, 07.85.Qe, 41.50.+h, 61.05.cf

\section{INTRODUCTION}

New-generation synchrotron radiation sources and $\mathrm{x}$-ray free-electron lasers are capable of delivering x-ray beams with unprecedented brilliance and coherence. Nowadays, many advanced experimental techniques, such as coherent x-ray diffraction, x-ray phase contrast imaging, and x-ray photon correlation spectroscopy, take advantage of the inherently high coherence of the x-ray beams [1]. However, the beam coherence is often degraded by optical components employed in the beamline [2]. Therefore, the push towards diffractionlimited and coherence-preserving beams demands simple and accurate measurement of the complex coherence function. The coherence measurement is more challenging for $\mathrm{x}$ rays than for visible light, and specially fabricated optical elements are usually required [3-7]. Visible-light and x-ray methods differ vastly in their experimental setup, optical components, and the nature of signal recorded. Some optics-free methods based on the study of diffraction pattern using well-defined apertures have also been proposed [8,9]. However, most of these techniques can provide transverse coherence information only along a single direction. To overcome this limitation, one-dimensional (1D) gratings have been extended to twodimensional (2D) gratings [10], and recently to circular gratings to measure the coherence length along transverse directions [11]. Recently, a more general approach using colloidal particles was also proposed to measure the coherence properties of the X-ray source [12]. However, these proposed techniques require visibility measurements at multiple distances to obtain a coherence map. The detector must travel across a distance of hundreds of millimeters up to several meters [13] depending upon the beam coherence, thereby

\footnotetext{
*hongchang.wang@diamond.ac.uk

Published by the American Physical Society under the terms of the Creative Commons Attribution 3.0 License. Further distribution of this work must maintain attribution to the author(s) and the published article's title, journal citation, and DOI.
}

making earlier approaches not only time consuming but also impractical to implement on many beamlines.

In this work, we propose and demonstrate how an alternative approach using near-field speckle can be used to quantitatively characterize the transverse coherence properties with a simple phase membrane. Unlike existing techniques, the proposed approach does not require any high-precision optical elements or visibility measurements at multiple distances, and therefore it can enable in situ and fast coherence measurements for existing and future synchrotron radiation sources.

\section{THEORY}

In general, the coherence properties are described by mutual coherence functions defined as correlations between the wave fields separated in space and time. For quasimonochromatic and quasistationary processes, it is convenient to use the normalized version of the mutual intensity function, known as the complex coherence function (CCF) [14,15],

$$
\gamma=\left\langle E\left(r_{1}\right) E^{*}\left(r_{2}\right)\right\rangle / \sqrt{I\left(r_{1}\right) I\left(r_{2}\right)}
$$

where $E$ and $I$ are the amplitude and intensity of the field at the points $r_{1}$ and $r_{2}$, respectively. The modulus of the $\mathrm{CCF}$ is directly related to the visibility of the fringes formed by inteference of two wave fronts [15], which is easily measurable. The approach proposed in this paper is based on the analysis of the near-field speckles from a statistical scatterer. The near-field speckle technique was proposed for measurement of dynamical properties of soft matter, colloids, and suspensions at the beginning of this century [16]. Though initially proposed with lasers, this method was shown to be equally applicable to $x$ rays [17]. One of the advantages with $\mathrm{x}$ rays is that the near-field regime extends to quite large distances, thereby making the experimental setup more flexible $[18,19]$. The maximum sample-to-detector distance for which the near-field condition (Fresnel condition) is satisfied can be defined as; $z=k \xi^{2}$, where $k$ is the wave number and $\xi$ is the coherence length. Therefore, for x-ray energy of $E=15 \mathrm{keV}$, this condition is easily satisfied up to the distance 
of a few meters $(\sim 7 \mathrm{~m})$, even if a moderate coherence length of $10 \mu \mathrm{m}$ is assumed. Let us consider near-field scattering from a static scatterer such as a membrane filter with a nominal pore size of several microns. The synchrotron radiation is partially coherent and therefore can be considered as being made up of a large number of coherent areas, which vary in both space and time. Any measurement on a longer time scale than the fluctuations and the lifetime of these coherent areas will be the superposition of many interference fringes from the same scatterer. As the synchrotron source's coherence length is at least an order of magnitude larger than the pore size, it is expected that a typical coherent area would encounter many of these pores distributed randomly, thereby giving a speckle pattern. Mathematically the recorded intensity at the detector can be described by following equation:

$$
\begin{aligned}
I(x, y) & =\left|a_{0}\right|^{2}+a_{0}^{*} a_{s}+a_{0} a_{s}^{*}+\left|a_{s}\right|^{2} \\
& \cong\left|a_{0}\right|^{2}+a_{0}^{*} a_{s}+a_{0} a_{s}^{*} \\
& \equiv I_{0}+2 \operatorname{Re}\left(a_{0}^{*} a_{s}\right),
\end{aligned}
$$

where $a_{0}$ and $a_{s}$ represent the incident and scattered beam amplitudes and it is assumed that $a_{s} \ll a_{0}$. The normalized scattered intensity distribution is obtained by subtracting the background intensity from the recorded speckle intensity pattern as follows:

$$
a_{n}=2 \operatorname{Re}\left(\frac{a_{s}}{a_{0}}\right)=\frac{I-I_{0}}{I_{0}} .
$$

As described earlier, the CCF can be deduced by analyzing the cross-correlation of the scattered signal. Although the analysis can be done in real space, it is more convenient to understand that various factors affect the visibility map in reciprocal space as they are related by the Wiener-Khintchine theorem. In the near-field regime, the power spectrum of the normalized scattered signal can be decomposed as [17]

$$
I(q)=S(q) I_{S}(q)
$$

where $S(q)$ is the near-field transfer function and $I_{S}(q)$ is the Fourier transform of the electronic density distribution of the membrane filter. It may be noted that there is a limit to the frequency range $\left(q_{\min }, q_{\max }\right)$ accessible by this method. While the highest frequency $q_{\max }$ for this procedure is generally limited by detector pixel size, often the low frequency $q_{\text {min }}$ is limited by instabilities of the beamline optics. The transfer function in the range $\left(q_{\min }, q_{\max }\right)$ is described by the following equation $[17,20]$ :

$$
S(q)=T_{\mathrm{tal}}(q) T_{\mathrm{coh}}(q) T_{\mathrm{det}}(q),
$$

where $T_{\mathrm{tal}}(q)$ is the distance-dependent Talbot effect, $T_{\text {coh }}(q)$ describes effects due to partial coherence of the incident wave front, and $T_{\text {det }}(q)$ describes the detector response function. The first two factors are fundamental in nature and related to the wave nature of radiation and the coherence properties of the source. The last factor can be determined experimentally and is associated with the data collection process. In the near-field region, $T_{\text {tal }}(q)$ introduces a $z$-dependent oscillatory dependence on the transfer function. It can be described as

$$
T_{\mathrm{tal}}(q)=\sin ^{2}\left(q^{2} z / 2 k\right)
$$
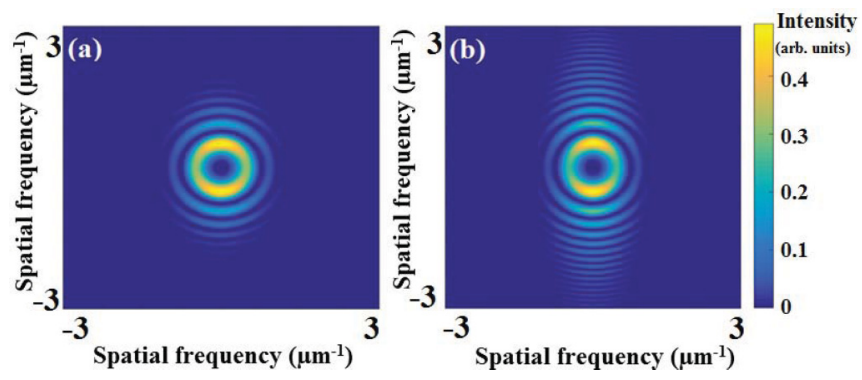

FIG. 1. (Color online) The power spectrum of near-field speckles for two different transverse coherence lengths (a) $\left(\xi_{x}=24 \mu \mathrm{m}\right.$, $\left.\xi_{y}=53 \mu \mathrm{m}\right)$ and (b) $\left(\xi_{x}=24 \mu \mathrm{m}, \xi_{y}=780 \mu \mathrm{m}\right)$. The near-field power spectrum shows dependence upon the coherence length.

where $z$ is the detector-to-membrane distance. $T_{\text {det }}(q)$, the detector's frequency response, includes effects of the scintillator screen and optics and is determined experimentally. The most conventional techniques are to place a knife edge near the detector in order to obtain its modulation transfer function or to feed the detector with a random white noise pattern. The effect of partial coherence on the transfer function can be evaluated by using the van Cittert and Zernike theorem. Assuming a Gaussian intensity distribution in the source plane, the coherence transfer function is described by the following relation:

$$
T_{\mathrm{coh}}(q)=\left[\exp \left(-\frac{x^{2}}{2 \xi_{x}^{2}}-\frac{y^{2}}{2 \xi_{y}^{2}}\right)\right]^{2},
$$

where $\xi_{x}$ and $\xi_{y}$ are transverse coherent lengths (rms) in the horizontal and vertical directions, respectively. As described by Eq. (4), the speckle power spectrum can be affected by variation in any of the product terms. Thus by choosing a scatterer whose power spectrum response is uniform in the accessible $q$ range, the effect of the transfer function can be easily studied. Importantly, the coherent properties of the source can be investigated fully in two dimensions, and effective $\mathrm{x}$-ray source sizes can be determined accordingly. It may be noted that that even in phase contrast imaging these effects are present, and special care needs to be taken to avoid the loss of information while retrieving the phase from the intensity measurements.

Figure 1 shows the simulated near-field speckle power spectrum for two different coherent length parameters. While the power spectrum of Fig. 1(a) is simulated with a typical bending magnet source $\left(\xi_{x}=24 \mu \mathrm{m}, \xi_{y}=53 \mu \mathrm{m}\right)$ at the Diamond light source, Fig. 1(b) is akin to the one from an undulator source $\left(\xi_{x}=24 \mu \mathrm{m}, \xi_{y}=780 \mu \mathrm{m}\right)$. For these illustrations, a detector pixel of $1 \mu \mathrm{m}$ and a membrane with $0.8 \mu \mathrm{m}$ pore size was assumed and the detector-to-membrane distance was taken as $100 \mathrm{~cm}$. The detector power spectrum was assumed to be of the form $T_{\operatorname{det}}(q)=\exp (-\alpha q)$ with $\alpha=1.25 \mu \mathrm{m}$.

The higher coherence along the vertical direction translates into Talbot fringes covering a larger frequency range in the power spectrum map. As seen from these figures, the near-field power spectrum map shows dependence on the coherent properties of the incident radiation and hence information about the coherent properties of radiation can be obtained 


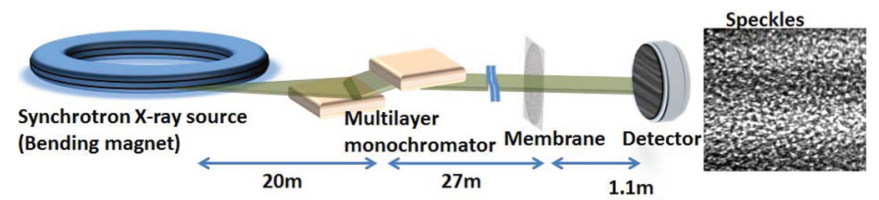

FIG. 2. (Color online) Schematic of the experimental arrangement for coherence measurements using near-field speckles.

from these measurements. One of the objectives of the present work is to show that the analysis of the speckle power spectrum collected at this single distance permits evaluation of the transverse coherence length along all directions in the transverse plane.

\section{EXPERIMENTS}

The principle of the technique presented here has been validated by measurements. The experiments were performed at the Diamond light source's B16 test beamline [21] using $\mathrm{x}$ rays from a bending magnet source.

\section{A. Speckle-based coherence measurement}

Figure 2 is the schematic of the experimental setup. A membrane with an average pore size of $0.8 \mu \mathrm{m}$ was mounted on a motorized stage at a distance of $47 \mathrm{~m}$ from the source. Images of the speckle pattern were collected using a high-resolution $\mathrm{x}$-ray microscope placed downstream of the membrane. The camera is based on the pco.4000 CCD detector, uses a Ce-doped YAG scintillator, and, with a $10 \times$ microscope objective, provides an effective pixel resolution of $0.9 \mu \mathrm{m}$. A double multilayer monochromator was used to select energy of $15 \mathrm{keV}\left(\triangle E / E \approx 10^{-2}\right)$ for these experiments. The estimated flux at the sample position in this configuration is about $1 \times 10^{11}$ photons $/ \mathrm{s} / \mathrm{mm}^{2}$, and the beam size is $2(\mathrm{H}) \times 4 \mathrm{~mm}^{2}$. The membrane (cellulose acetate) of $0.8 \mu \mathrm{m}$ pore was mounted on the multitranslation motorized stage that allows easy alignment and scanning of the membrane.

Figure 3(a) shows a typical raw speckle spectrum collected at $0.16 \%$ electron beam coupling conditions. As seen, the horizontal stripes are due to the multilayer monochromator used for collecting the images and its effect can be seen from the power spectrum.

In principle, only a single image needs to be acquired for this technique. However, in order to improve signal-to-noise

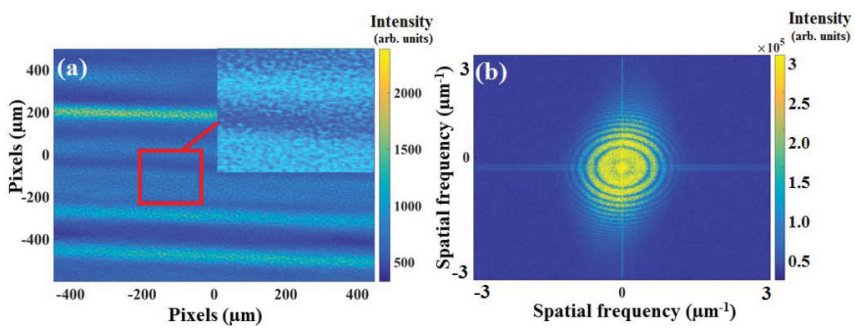

FIG. 3. (Color online) Images of (a) raw speckle pattern and (b) its power spectrum at $0.16 \%$ coupling conditions. The power spectrum has low-frequency features mostly due to the stripes of the multilayer monochromator. The inset shows a magnified image of the raw speckle pattern. ratio and remove contributions of stray signal, detector noise, etc. the data were collected by raster scanning the membrane stage $\left[0.5(\mathrm{H}) \times 0.5(\mathrm{~V}) \mathrm{mm}^{2}\right]$ and generating a multiple data set $[20(\mathrm{H}) \times 20(\mathrm{~V})]$ of the same region of interest of the membrane. Data acquisition time was $1 \mathrm{~s}$ for each image. The data set was cropped to $200 \times 200$ pixels and the correction for the background data is achieved by averaging of all the independent scans. The average consists of all the terms such as stray signal and dark-noise from the detector which remains constant in the entire independent speckle pattern.

Once the raw data are generated and background is properly removed, the 2D power spectrum of the near-field speckle is obtained. The detector transfer function was evaluated separately and was used as an input parameter during the analysis. The was then fitted to following equation:

$$
I(q)=a_{1}|S(q)|^{2}+a_{2},
$$

where $a_{1}$ and $a_{2}$ represent the fitting parameters. The detector response in the frequency space was modeled as $\exp (-\alpha q)$ where $\alpha$ is evaluated experimentally. The data are processed in two steps. First the detector response function was evaluated by keeping the membrane in close proximity with the detector and scanning the membrane as described previously. We use the measured sample-to-detector distance and calculate the detector response function that fits for all the different values of distances $(z=10 \sim 30 \mathrm{~mm})$. The value for $\alpha=1.25 \mu \mathrm{m}$ was obtained using this process and was used for deriving coherence properties of the source. The angular power spectrum is then fitted with a near-field transfer function as described in Eq. (8) for each angular position, using a nonlinear least-square fitting procedure, as illustrated in Fig. 4. This process treats the overall amplitude and coherence length as fitting parameters and the measured near-field spectrum and membrane-to-detector distance were used as input.

Importantly, a rare access to the Diamond storage ring was obtained when the vertical coupling of the electron beam in the storage ring was varied to verify the proposed technique. The measurements were carried out by changing the vertical electron-beam coupling in the $0.16 \%-1.33 \%$ range to facilitate the experiment at different vertical source sizes. Figure 5 shows the power spectrum of the speckle at two different coupling conditions. The fringes observed in

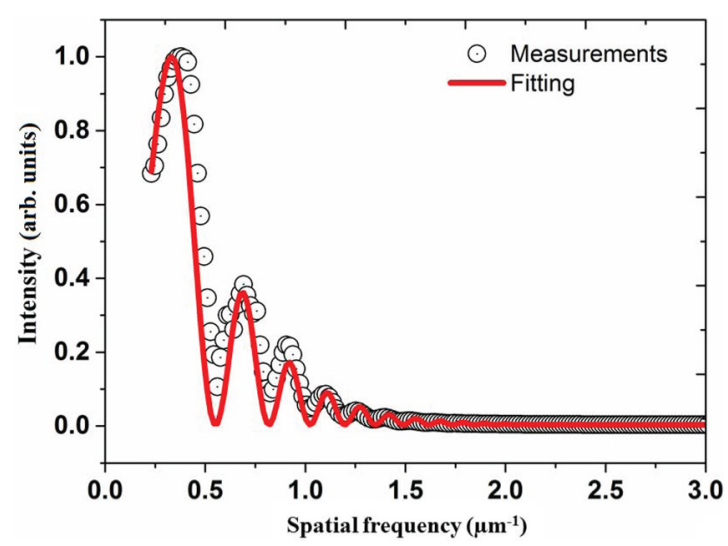

FIG. 4. (Color online) The experimental power spectrum along with fitted spectrum as a function of spatial frequency. 

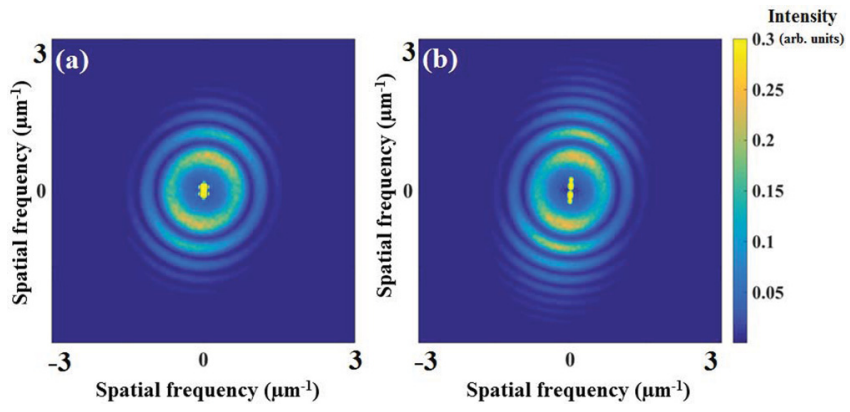

FIG. 5. (Color online) Power spectrum of the speckle in (a) $1.33 \%$ and (b) $0.16 \%$ coupling conditions. The power spectrum map shows that the coherence length in the vertical direction in low-coupling conditions is higher as compared to its value at higher coupling, while in the horizontal direction it remains unchanged.

the power spectrum in low-coupling mode $(0.16 \%)$ extend to much higher frequencies in the vertical direction than in high-coupling mode $(1.33 \%)$, thereby verifying that only vertical source size is affected.

Following the above procedure, the retrieved twodimensional coherence lengths for the vertical electron-beam coupling at $0.16 \%, 0.31 \%, 0.66 \%, 1.00 \%$, and $1.33 \%$ are shown in Fig. 6. As expected, the vertical coherence length increases when the vertical coupling is decreased, while the horizontal coherence length remains nearly the same. In addition, it can be seen that the ellipse of the coherence map is slightly tilted clockwise, while the tilting angle is the same for all the vertical coupling modes. These results also confirm that the electron beam does not get deformed by varying the vertical coupling. This capability to access the two-dimensional transverse coherence map may be of crucial importance for understanding the source properties, especially for insertion-device-based beamlines. Further studies showed that the observed tilt is mainly due to misalignment of the optical axes of the scintillator and CCD camera. Once the coherence length is derived, the corresponding source size was then calculated using the relation $s=2.35 \lambda R / 2 \pi \xi$, where $\lambda$ is the X-ray wavelength and $R$ is the phase membrane distance from the source. The calculated and measured source sizes with the speckle technique for the five vertical coupling modes are tabulated in Table I.

In addition, the real-time measurements using a pinhole camera [22] are also included for comparison. It can be seen that the horizontal source size does not change with a change in vertical coupling, and that the speckle and pinhole measurements match very well. As shown in Table I, the

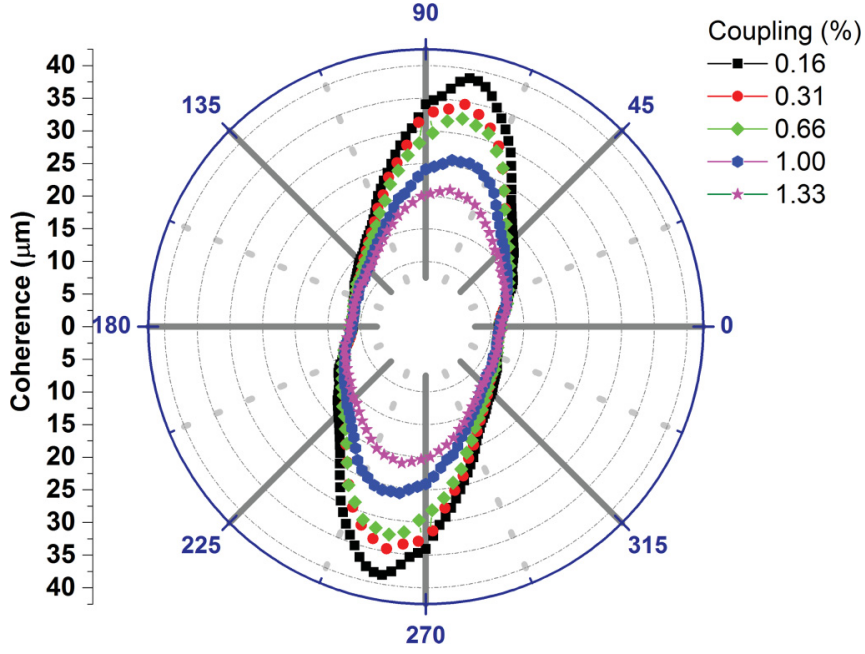

FIG. 6. (Color online) Two-dimensional transverse coherence distribution obtained using the speckle technique. The data were fitted with a tilted ellipse to obtain the horizontal and vertical coherence lengths.

vertical source size measurements using the speckle technique are in good agreement with the ones from the pinhole camera method at higher coupling modes.

The deviation of the source size measurements at low coupling values indicates that reduction in the coupling does not translate into corresponding improvements in the beam coherence at the beamline end station. This may be partially due to the imperfection of beamline optics and the vibrations induced by the beamline components. This conclusion is further confirmed by independent measurements using x-ray grating interferometery.

\section{B. Comparison with grating interferometry}

In order to further validate the speckle measurement results, coherence measurements using $\mathrm{x}$-ray grating interferometery were carried out [6] at two different electron-beam vertical coupling values, $0.3 \%$ and $1.0 \%$. The pitch of the phase grating was $4 \mu \mathrm{m}$ while that of the analyzer grating was $2 \mu \mathrm{m}$. These gratings were designed for use at $15 \mathrm{keV}$ and were aligned so as to generate Moiré fringes with sufficient contrast for further data processing. A fiber-optics CCD camera with pixel size of $6.4 \mu \mathrm{m}$ was used to collect the image. Data were collected for $0.5 \mathrm{~s}$ at each intergrating distance. The first grating was mounted on the sample stage at $R=47 \mathrm{~m}$ from the source and the absorption grating was located downstream of the

TABLE I. Measured source size (FWHM) in horizontal and vertical directions using near-field speckles and vertical source size using a pinhole camera.

\begin{tabular}{|c|c|c|c|c|}
\hline \multirow[b]{2}{*}{ Coupling (\%) } & \multicolumn{2}{|c|}{ Speckle technique } & \multicolumn{2}{|c|}{ Pinhole camera } \\
\hline & Horizontal $(\mu \mathbf{m})$ & Vertical $(\mu \mathbf{m})$ & Horizontal $(\mu \mathbf{m})$ & Vertical $(\mu \mathbf{m})$ \\
\hline 0.16 & 120 & 38 & 121 & 25 \\
\hline 0.31 & 123 & 42 & 122 & 34 \\
\hline 0.60 & 123 & 53 & 123 & 47 \\
\hline 1.00 & 125 & 63 & 123 & 60 \\
\hline 1.33 & 125 & 72 & 124 & 68 \\
\hline
\end{tabular}


(a)

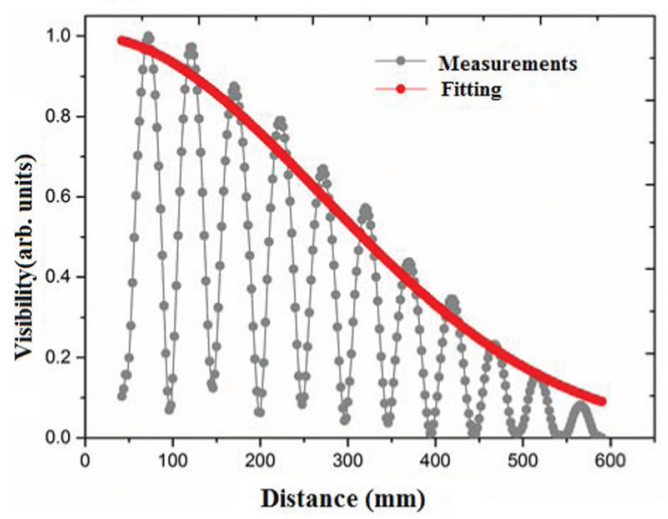

(b)

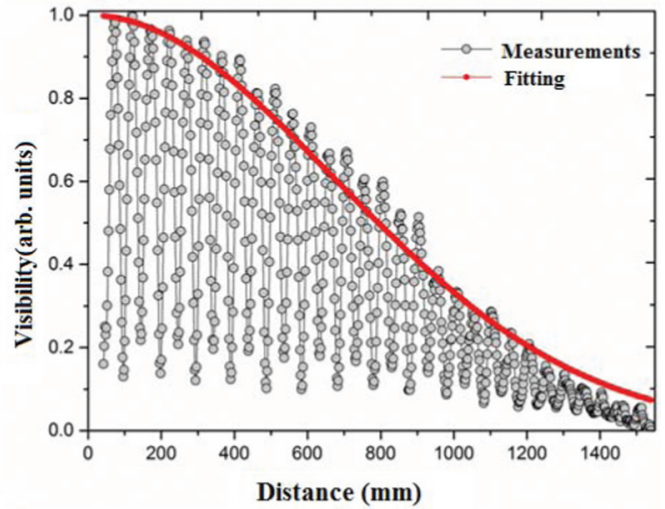

FIG. 7. (Color online) (a) Horizontal and (b) vertical coherence measurements using the visibility of Moiré fringes produced by grating interferometers as a function of distance between the two gratings at $0.3 \%$ coupling.

phase grating, in close contact with the detector. The distance between the gratings was increased in steps of $2 \mathrm{~mm}$ up to the maximum permissible distance limit allowed by the experimental setup. For each image of the scan, the visibility was calculated. The visibility curve (Fig. 7) was then fitted with a function defined by $A \exp \left(-z^{2} / 2 \sigma^{2}\right) \cos (2 \pi z / B+C)$, where the parameters $A, B, C$, and $\sigma$ were obtained by fitting the experimental data set as a function of intergrating separation $z$. The calculation of the coherence length was then done using the formula $\xi=2 \lambda \sigma / p$ and the corresponding source size was calculated using $s=2.35 \sigma_{s}=2.35 \lambda R / 2 \pi \xi$, where $\lambda$ is the $\mathrm{x}$-ray wavelength $p$ is the period of the phase grating, and $R$ is the phase grating distance from the source.

The horizontal coherence length obtained for these measurements was $11 \mu \mathrm{m}$ for both coupling conditions, thereby giving the horizontal source size 127-130 $\mu \mathrm{m}$. The vertical coherence length was found to be 31 and $25 \mu \mathrm{m}$ for $0.3 \%$ and $1.0 \%$ coupling, respectively, and calculated source sizes were 46 and $58 \mu \mathrm{m}$, respectively. As shown in Table I, the corresponding results for the source size using the speckle technique were 42 and $63 \mu \mathrm{m}$, respectively. Thus these results confirm that beamline optics degrades the coherence properties of the beam, thereby increasing the effective source size in the vertical direction.

\section{SUMMARY}

In conclusion, we have shown that near-field speckle can be used to generate full two-dimensional coherence maps and derive the source size. This technique is model-free and easy to implement, and information along all transverse directions can be obtained in a single distance measurement. The technique is expected to be very useful for measurement of transverse coherence and its degradation by optical elements along any direction. The phase membrane can be replaced with colloids, thereby utilizing the Brownian particle motion to remove the mechanical movements of the phase membrane. The present approach is easy to implement and is expected to find wide applications in measurements of coherence properties of current and future synchrotron-based x-ray sources.

\section{ACKNOWLEDGMENTS}

This work was carried out with the support of Diamond Light Source Ltd. The authors are grateful to John Sutter for correcting the manuscript and to Ian Pape and Andrew Malandain for help during the experiments. We are also extremely grateful to the Diamond technical division, in particular to Riccardo Bartolini and Vince Kempson for facilitating electron-beam coupling changes in the storage ring.
[1] Third Generation Hard X-ray Synchrotron Radiation Sources, edited by D. Mills (John Wiley and Sons., New York, 2002).

[2] I. A. Vartanyants and I. K. Robinsons, Opt. Commun. 222, 29 (2003).

[3] J. J. A. Lin et al., Phys. Rev. Lett. 90, 074801 (2003).

[4] D. Paterson et al., Opt. Commun. 195, 79 (2001).

[5] W. Leitenberger et al., J. Synchrotron Radiat. 11, 190 (2004).

[6] F. Pfeiffer, O. Bunk, C. Schulze-Briese, A. Diaz, T. Weitkamp, C. David, J. F. van der Veen, I. Vartanyants, and I. K. Robinson, Phys. Rev. Lett. 94, 164801 (2005).

[7] A. Snigirev, I. Snigireva, V. Kohn, V. Yunkin, S. Kuznetsov, M. B. Grigoriev, T. Roth, G. Vaughan, and C. Detlefs, Phys. Rev. Lett. 103, 064801 (2009).
[8] A. Snigirev et al., Rev. Sci. Instrum. 66, 5486 (1995).

[9] V. Kohn, I. Snigireva, and A. Snigirev, Phys. Rev. Lett. 85, 2745 (2000).

[10] Shashidhare Marathe et al., Opt. Express 22, 14041 (2014).

[11] X. Shi, S. Marathe, M. J. Wojcik, N. G. Kujala, A. T. Macrander, and L. Assoufid, Appl. Phys. Lett. 105, 041116 (2014).

[12] M. D. Alaimo, M. A. C. Potenza, M. Manfredda, G. Geloni, M. Sztucki, T. Narayanan, and M. Giglio, Phys. Rev. Lett. 103, 194805 (2009).

[13] C. Gutt et al., Phys. Rev. Lett. 108, 024801 (2012).

[14] L. Mandel and E. Wolf, Optical Coherence and Quantum Optics (Cambridge University Press, Cambridge, 1995). 
[15] M. Born and E. Wolf, Principles of Optics (Pergamon Press, Oxford, 1980).

[16] M. Giglio, M. Carpineti, and A. Vailati, Phys. Rev. Lett. 85, 1416 (2000).

[17] R. Cerbino et al., Nat. Phys. 4, 238 (2008).

[18] S. Berujon, H. Wang, and K. Sawhney, Phys. Rev. A 86, 063813 (2012).

[19] S. Berujon, E. Ziegler, R. Cerbino, and L. Peverini, Phys. Rev. Lett. 108, 158102 (2012).
[20] J. W. Goodman, in Laser Speckle and Related Phenomenon, edited by J. D. Dainty (Springer-Verlag, Berlin, Heidelberg, 1975).

[21] K. J. S. Sawhney et al., in SRI 2009: Tenth International Conference on Radiation Instrumentation, edited by R. Garrett, I. Gentle, K. Nugent, and S. Wilkins, AIP Conf. Proc. No. 1234 (AIP, New York, 2010), p. 387.

[22] C. Thomas, G. Rehm, I. Martin, and R. Bartolini, Phys. Rev. ST Accel. and Beams 13, 022805 (2010). 